\title{
Multiple sclerosis caused by drug-induced hepatitis: A rare case report
}

\author{
Rodrigo Marmo da Costa e Souza ${ }^{1,2}$ and Thiago Monteiro de Paiva Fernandes ${ }^{1 *}$ \\ ${ }^{1}$ Cognitive Neuroscience and Behavior Program, Federal University of Paraiba, Joao Pessoa, Brazil \\ ${ }^{2}$ Chief and Surgeon, Neurology Department, Emergency and Trauma Hospital, Joao Pessoa, Brazil
}

\begin{abstract}
A 51-year-old white Brazilian female woman initially presented symptoms similar to migraine and chronic pain after the birth of her second child. After confusing diagnosis and continuous use of painkillers, the patient presented drug-induced hepatitis. A complete neurological evaluation was performed, where the imaging tests proved the existence of multiple sclerosis (MS). According to our knowledge, this is the first reported case in the literature. Thus, our study highlights the importance of alerting physicians in closely observing the variables presented in each neurological case.
\end{abstract}

\section{Background}

Multiple sclerosis (MS) is a demyelinating disease of the central nervous system, having as factors of association inflammation, degeneration and autoimmune processes [1]. Early diagnosis of MS is still a challenge and depends on detailed clinical analysis, excluding other neurological diseases with similar characteristics $[2,3]$.

Here we describe the case of a 51-year-old woman who initially presented similar symptoms to migraine and chronic pain after the birth of her second child. After confusing diagnosis and continuous use of painkillers, the patient presented drug-induced hepatitis. A complete neurological evaluation was performed, where the imaging tests proved the existence of MS. According to our knowledge, this is the first reported case in the literature.

\section{Case presentation}

A 51-year-old white Brazilian woman began to experience severe pain in the head and forehead after the birth of her second child. In a clinical interview, the patient reported absence of visual symptoms and according to the International Headache Society criteria (ICHDIII) [4], the patient was diagnosed with migraine without aura, and nortriptyline $(25 \mathrm{mg} /$ day) was prescribed. The patient discontinued the medication spontaneously after seven months, complaining of notable weight gain and irritability. After continuing experiencing severe pain in the head and forehead region, the patient began to use Cefaliv (3 times/day).

In a new clinical interview, the patient reported that the headaches began after the birth of her second child and increased after reaching the age of 45 years. No family history of migraine, neuropsychiatric disorders, substance abuse has been reported. Neurological examination indicated slight impairments in information processing speeds. Ophthalmologic examination indicated intraocular pressure of $17.09 \pm 2.25$ and no abnormalities were observed in the specular corneal microscopy exam. The patient reported no symptoms such as photosensitivity or epileptic seizures, but mentioned that a complete battery of exams had been performed previously. Conventional electroencephalography (EEG) and magnetic resonance imaging (MRI) studies comprising T1, T2 and Flair (Figure 1) were performed.

Examination of EEG indicated epileptiform activity in outbreaks in temporal areas of the right hemisphere. Her physician denied clinical significance for imaging tests a performed a susceptibility weighted imaging (SWI) (Figure 2).

During the following queries, the patient complained experiencing depressive symptoms and agoraphobia. She reports that her physician observed the new imaging tests and noted that there was also no clinical significance. Considering the psychological symptoms comorbidities of migraine, the patient was prescribed paroxetine (50 $\mathrm{mg}$ /day). However, the patient noticed continuity of psychological and physical symptoms. About a year using the same medication, the patient presented a discomfort followed by pain in the abdomen. In new neurological examination, abdominal MRI was performed and drug-induced hepatitis was observed (Figure 3).

In a new neurological evaluation, the neurosurgeon indicated that the MRI results for T1 and T2 really did not indicate clinical significance. However, SWI showed calcification of the basal ganglia, and in Flair a slight microangiopathy was observed in the frontal horn.

Based on her medical history, it was hypothesized that she may have developed choric pain or multiple sclerosis after a possible postpartum depression. Since no exams were performed right after the birth of her child, and considering the elimination of misdiagnoses, the patient was

Correspondence to: Thiago M. de Paiva Fernandes, Departamento de Psicologia, CCHLA - UFPB. Campus I, Cidade Universitária, CEP: 58051-90, João Pessoa PB; Brazil, E-mail: thiagompfernandes@gmail.com

Key words: multiple sclerosis, chronic pain, neurological assessment, magnetic resonance imaging, case report

Received: April 22, 2017; Accepted: May 26, 2017; Published: May 30, 2017 


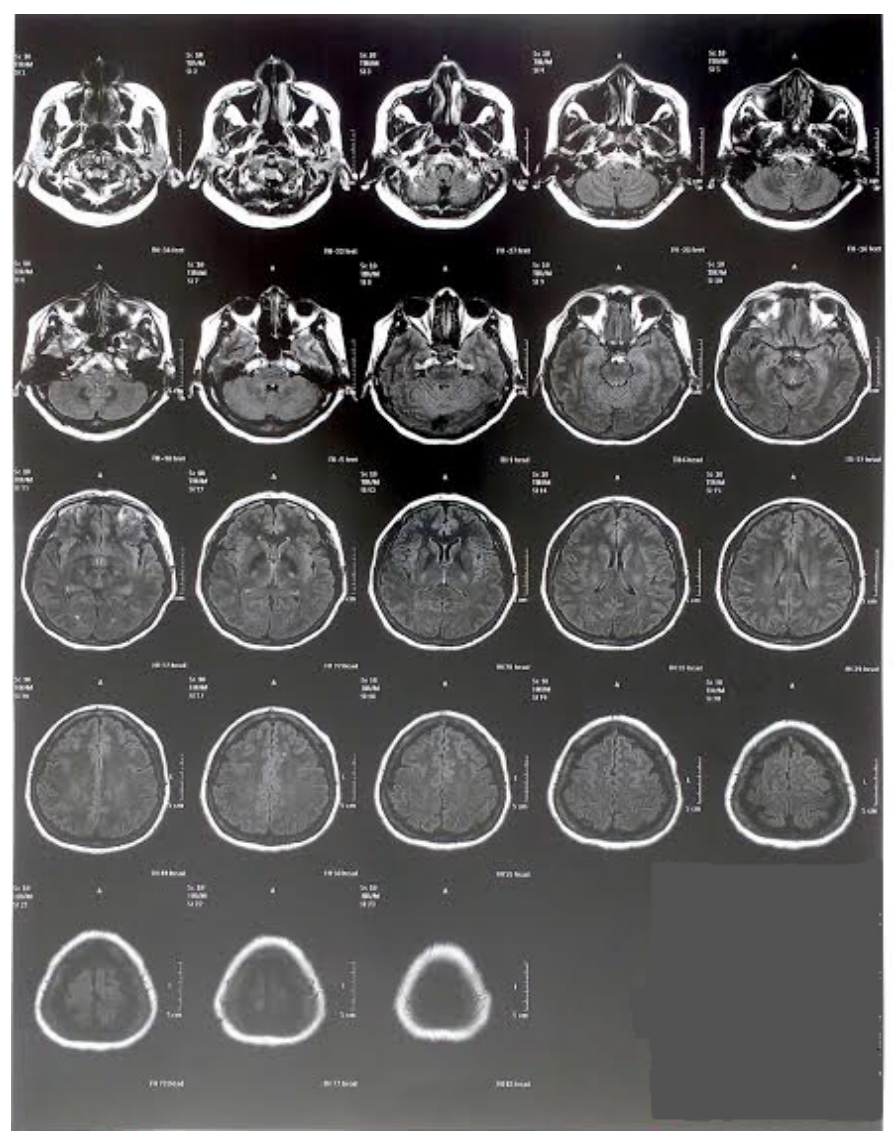

Figure 1. Flair MRI showing microangiopathy in the frontal horn.

diagnosed with multiple sclerosis caused by drug-induced hepatitis.

After treatment of hepatitis, gabapentin (100mg) 3 times daily was prescribed. There was no response. The dose was increased to $300 \mathrm{mg}$ 3 times daily. The patient will continue to follow up to observe the progress of the case.

\section{Discussion}

Multiple sclerosis (MS) is a demyelinating disorder of the central nervous system [1], and a common cause of disability in adults [5]. The occurrence of axonal damages may result in neuropathic pain, which makes it challenge to early diagnose MS. In the absence of disability, even in the earliest stages of disease evolution, the symptoms of MS and migraine may complicate an accurate diagnosis [6,7]. It is known that headaches and migraine are common in MS [7], but the absence of other diagnostic criteria for MS prevented this disorder from being detected early. In addition, given the inability to correlate the onset of MS after postpartum depression, the continued use of medications for more than 25 years was yet another factor that confounded physicians during this time.

Neurophysiological mechanisms involving migraine and MS are not completely understood $[8,9]$, but there are some signs that may be observed as the idiopathic basal ganglia calcification [10], chronic fatigue [11], and the observation of the genesis and progression of microangiopathy $[12,13]$.

Reduction in information processing speed [14] and executive functions [15] as well as increasing in intraocular pressure are indicative of MS [16]. However, to correlate these variables to MS, a

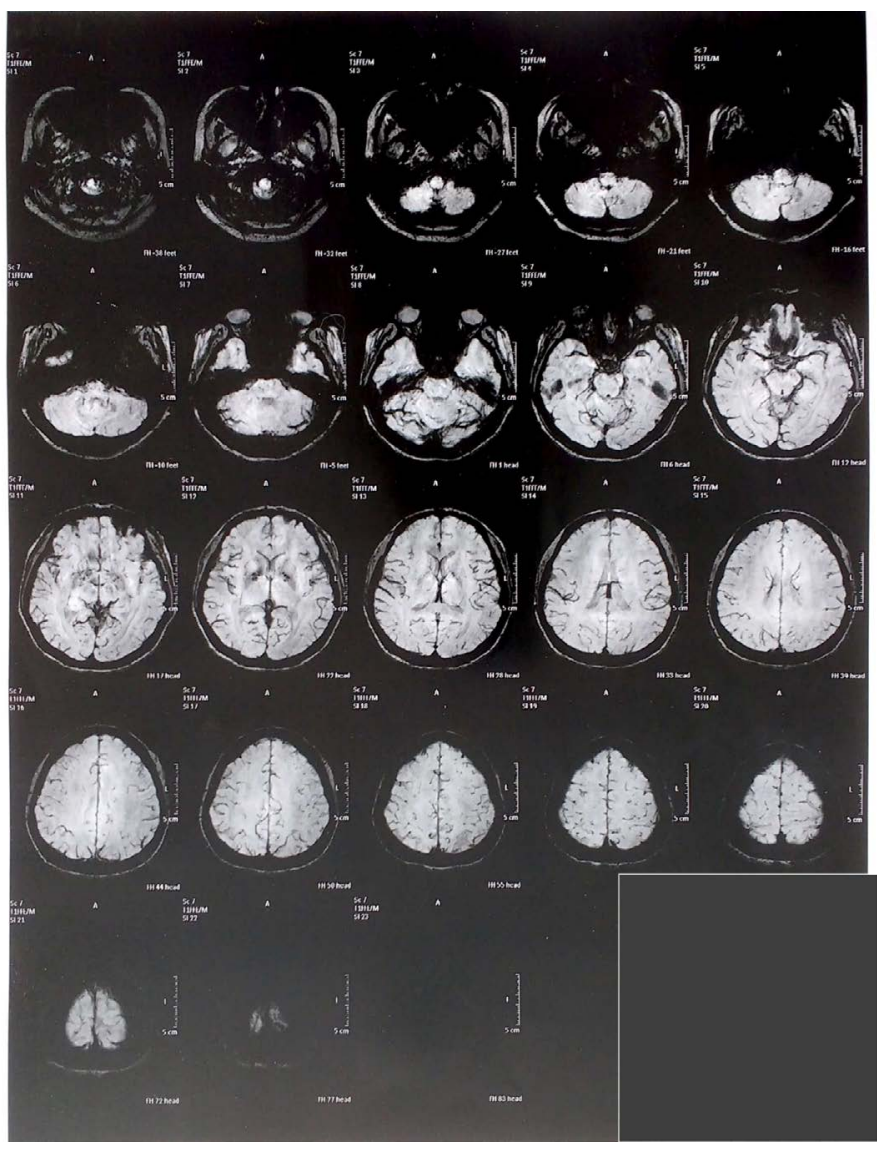

Figure 2. Susceptibility weighted imaging showing basal ganglia calcification.

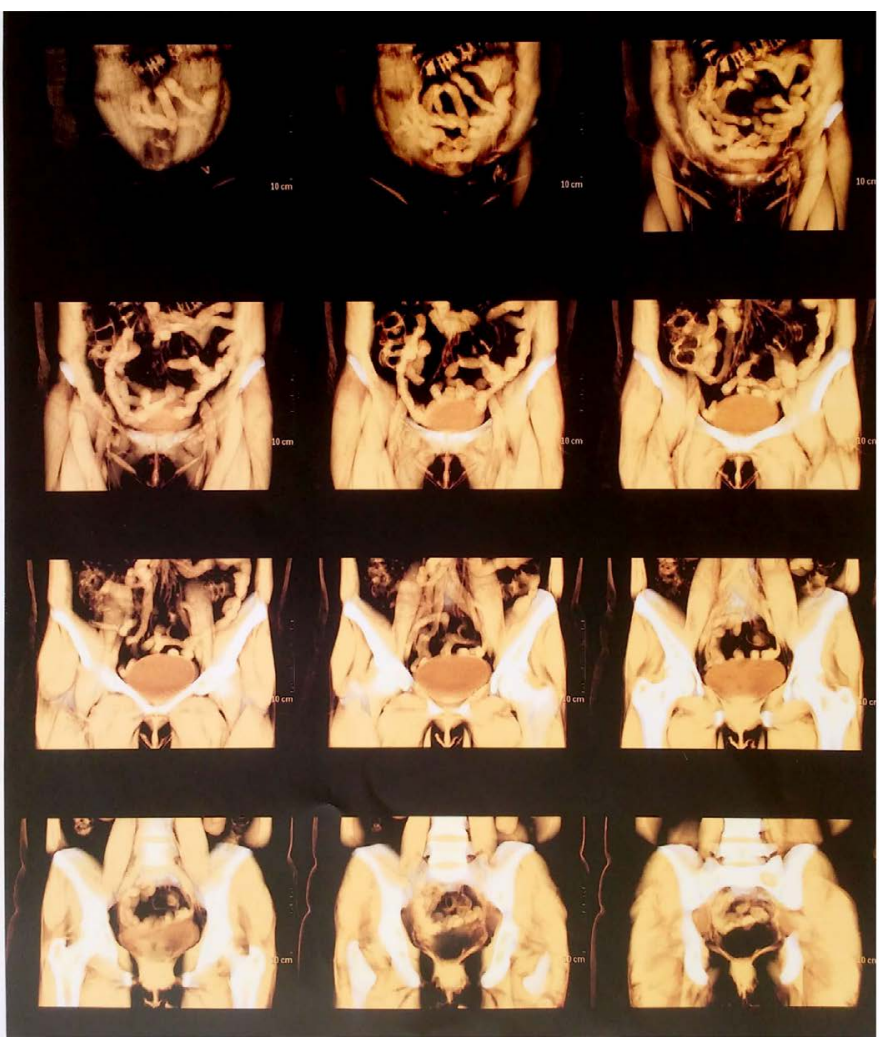

Figure 3. Abdominal MRI with contrast showing drug-induced hepatitis. 
detailed analysis of the whole case would be required, which did not occur for approximately 25 years.

The use of drugs such as beta-blockers, tricyclic antidepressants or analgesics were not helpful in the treatment of her condition. This is another factor line in the confusion of the diagnosis. The presence of chronic pain can be confused with migraine [17] and is a characteristic of MS $[1,8]$. Thus, chronic use of these drugs, responsible for druginduced hepatitis, may have masked MS for a long time.

Our patient will continue to experience MS symptoms, since it is an incurable condition. Despite her current treatment, studies have shown the efficacy of furosemide for numerous conditions $[18,19]$. Further studies are necessary to observe and confirm the efficacy of furosemide in MS. However, we believe that the correct diagnosis will help her quality of life and promote a better prognosis.

There are few cases that report MS with postpartum depression [20], which also contributes to the difficulty of establishing diagnostic criteria for rare cases such as these. A detailed observation is requested in cases of postpartum depression and in cases of migraine where there are damages such as calcification or microangiopathy.

\section{Conclusions}

Although we do not know if the genesis of MS in this case was due to postpartum depression or drug-induced hepatitis, according to our knowledge, this is the first case of MS caused by drug-induced hepatitis.

Even considering that all cases were not similar, prior knowledge about the symptoms or characteristics of MS, as well as possible relationships with postpartum depression will improve diagnosis and prognosis. Thus, our study highlights the importance of alerting physicians in closely observing the variables presented in each neurological case.

\section{Declarations}

\section{Consent for publication}

Written informed consent was obtained from the patient for publication of this case report and any accompanying images. A copy of the written consent is available for review by the Editor-in-Chief of this journal.

\section{Availability of data and materials}

Not applicable

\section{Authors' contributions}

GH examined the case and assisted in the follow-up review. RM examined the case, was responsible for the direction and guidance of this case. TM performed the critical review of the case and was a major contributor in writing the manuscript. All authors read and approved the final manuscript

\section{Acknowledgements}

Not applicable

\section{Ethics approval and consent to participate}

The study was exempted Committee of Ethics in Research of the Health Sciences Center of Federal University da Paraiba.

\section{Competing interests}

The authors declare that the research was conducted in the absence of any commercial or financial relationships that could be construed as a potential conflict of interest.

\section{Funding}

\section{Not applicable}

\section{References}

1. Goldenberg MM (2012) Multiple sclerosis review. P T 37: 175-184. [Crossref]

2. Miller JR (2004) The importance of early diagnosis of multiple sclerosis. J Manag Care Pharm 10: S4-11. [Crossref]

3. Noyes K, Weinstock-Guttman B (2013) Impact of diagnosis and early treatment on the course of multiple sclerosis. Am J Manag Care 19: s321-331. [Crossref]

4. Headache Classification Committee of the International Headache Society (IHS) (2013) The International Classification of Headache Disorders, 3rd edition (beta version). Cephalalgia 33: 629-808. [Crossref]

5. Confavreux C, Vukusic S, Moreau T, Adeleine P (2000) Relapses and progression of disability in multiple sclerosis. N Engl J Med 343: 1430-1438. [Crossref]

6. Elliott DG (2007) Migraine in multiple sclerosis. Int Rev Neurobiol 79: 281-302. [Crossref]

7. Evans RW, Rolak LA (2001) Migraine Versus Multiple Sclerosis. Headache J Head Face Pain 41: 97-98

8. Hauser SL, Oksenberg JR (2006) The neurobiology of multiple sclerosis: genes, inflammation, and neurodegeneration. Neuron 52: 61-76. [Crossref]

9. Leocani L, Comi G (2014) Clinical neurophysiology of multiple sclerosis. Handb Clin Neurol 122: 671-679. [Crossref]

10. Abedini M, Karimi N, Tabrizi N (2015) Cooccurrence of Multiple Sclerosis and Idiopathic Basal Ganglia Calcification. Case Rep Med 2015: 838243. [Crossref]

11. Sandyk R, Awerbuch GI (1994) Pineal calcification and its relationship to the fatigue of multiple sclerosis. Int J Neurosci 74: 95-103. [Crossref]

12. O'Halloran HS, Pearson PA, Lee WB, Susac JO, Berger JR (1998) Microangiopathy of the brain, retina, and cochlea (Susac syndrome). A report of five cases and a review of the literature. Ophthalmology 105: 1038-1044. [Crossref]

13. Perlmutter LS, Chui HC (1990) Microangiopathy, the vascular basement membrane and Alzheimer's disease: a review. Brain Res Bull 24: 677-686. [Crossref]

14. Rao SM, St Aubin-Faubert P, Leo GJ (1989) Information processing speed in patients with multiple sclerosis. J Clin Exp Neuropsychol 11: 471-477. [Crossref]

15. Jongen PJ, Ter Horst AT, Brands AM (2012) Cognitive impairment in multiple sclerosis. Minerva Med 103: 73-96. [Crossref]

16. Akçam HT, Capraz IY, Aktas Z, Batur Caglayan HZ, Ozhan Oktar S, et al. (2014) Multiple sclerosis and optic nerve: an analysis of retinal nerve fiber layer thickness and color Doppler imaging parameters. Eye 28: 1206-1211. [Crossref]

17. Buse DC, Manack AN, Fanning KM, Serrano D, Reed ML, et al. (2012) Chronic migraine prevalence, disability, and sociodemographic factors: results from the American Migraine Prevalence and Prevention Study. Headache 52: 1456-1470. [Crossref]

18. de Almeida RF, Leão IA, Gomes JB, da Silva AA Jr, Teixeira AL (2009) Migraine with persistent visual aura: response to furosemide. Clinics (Sao Paulo) 64: 375-376. [Crossref]

19. Kallet RH (2007) The role of inhaled opioids and furosemide for the treatment of dyspnea. Respir Care 52: 900-910. [Crossref]

20. Akkaya C, Kocagoz SZ, Turan OF, Taskapilioglu O, Kirli S (2007) Onset of multiple sclerosis following post-partum depressive and manic episodes. Psychiatry Clin Neurosci 61: 698-699. [Crossref]

Copyright: (C2017 de Paiva Fernandes TM. This is an open-access article distributed under the terms of the Creative Commons Attribution License, which permits unrestricted use, distribution, and reproduction in any medium, provided the original author and source are credited. 